\title{
CLOSE-BINARY AND PULSATING CENTRAL STARS
}

\author{
Howard E. Bond \\ Space Telescope Science Institute \\ 3700 San Martin Drive \\ Baltimore, Maryland 21218 USA
}

\begin{abstract}
As a result of photometric-monitoring studies, 7 planetary-nebula nuclei are now known to be binaries with orbital periods less than one day. These systems were probably produced via a common-envelope interaction, during which a wide pair was converted to a close binary surrounded by an ejected red-giant envelope. The frequency of occurrence of such close binaries among PNNs is about $10-15 \%$, showing that binary-star interactions are a significant production mechanism for planetary nebulae. The descendants of close-binary PNNs are probably the cataclysmic variables. Two CVs surrounded by nebulae resembling old planetaries, $0623+71$ and GK Per, may provide the most direct evidence for the origin of CVs through PN ejection. The observed birth rate for close-binary PNNs is more than an order of magnitude higher than for CVs, possibly indicating that our census of the CV population is very incomplete. The nucleus of K 1-16 is a member of the GW Vir class of extremely hot pulsating pre-white dwarfs, and the only one known to be surrounded by a PN. These objects offer exciting opportunities for direct measurement of evolutionary timescales and for seismological investigations of the interiors of PNNs and their immediate descendants.
\end{abstract}

\section{LIGHT VARIABILITY AMONG PNNs}

This paper will review recent work on two classes of variable planetary-nebula nuclei (PNNs): (1) close binaries, in which the observed brightness of the system varies because of heating effects or actual eclipses; and (2) pulsating variables, in which a pulsational instability modulates the luminosity of the (single) central star. Over the past several years, several close-binary PNNs and one pulsating PNN have been discovered. Both classes of objects potentially offer a wealth of new information on the origin of planetary nebulae and on the evolution of their central stars.

\section{SEARCHES FOR CLOSE-BINARY PNNs}

The first close-binary central star, that of Abell 63, was actually discovered more than half a century ago by Hoffleit (1932). This suspected eclipsing binary received the variable-star designation UU Sge, but its faint surrounding PN 
was only discovered much later by Abell (1966); the coincidence of the objects was not noticed until another decade had passed (Bond 1976). Photoelectric photometry (Miller, Krzeminski, and Priedhorsky 1976; Bond, Liller, and Mannery 1978) established a remarkably short orbital period of $11^{\mathrm{h}} 10^{\mathrm{m}}$, showed that the eclipses are total, and demonstrated a strong "reflection effect" (heating of one hemisphere of the $\mathrm{dK}$ companion due to irradiation from the hot primary).

The existence of close-binary PNNs was predicted by Paczynski (1976), who proposed a scenario for the origin of compact binaries (such as the cataclysmic variables; see also Ritter 1976). The scenario involves an engulfed mainsequence companion spiralling down inside a red-giant envelope and ultimately spinning the common envelope up to breakup. This interaction would produce a close binary (the red-giant core plus the main-sequence secondary), lying inside a nebular shell that would be ionized by UV radiation from the hot core. The subsequent evolution of such a system would involve dissipation of the nebula, contraction of the hot core to white-dwarf dimensions, and, ultimately, contraction of the orbit and initiation of mass transfer due to magnetic braking (Verbunt and Zwaan 1981) or other mechanisms for loss of angular momentum.

The relative frequency of occurrence of binary vs. single PNNs would indicate the relative importance of binary-star v8. single-star PN ejection mechanisms. Moreover, the discovery of additional close-binary PNNs would provide unique opportunities for determination of fundamental properties of the central stars.

This review will concentrate on binary PNNs with $P<1$ day; these objects are the easiest to discover during photometric runs taken during one night, and it is these objects that are the probable progenitors of the cataclysmic variables (CVs). An accompanying paper in these proceedings by Méndez covers additional aspects of PNN binarity. At present, seven PNNs are known to show periodic photometric variations due to orbital motion in binaries with $P<1$ day. These objects are listed in Table I.

The table indicates whether the binaries show actual eclipses (UU Sge, V477 Lyr), or are variable only because of the reflection effect on the secondary stars. (Actually, our photometry of the southern object VW Pyx is not yet sufficient to rule out eclipses.) Six of the stars were described by Bond and Grauer (1987a), so few details will be given here; the references to Table I may be consulted for further information.

A seventh object, the central star of Abell 35, is included in Table I. Shortterm, low-amplitude variability was first discovered by Bond and Grauer (see Bond 1985). This variability has been found to be periodic by Jasniewicz (1987). His data are not yet sufficient to distinguish between the two possible periods listed in the table, but it seems plausible that the variations are due to a heated hemisphere on the cooler star in the system. The nucleus of Abell 35 is the only binary in Table I whose optical spectrum is dominated by the late-type component, which was classified G8 III-IV by Jacoby (1981). IUE observations, however, have revealed the extremely hot companion that is responsible for ionizing the nebula (Jacoby, private communication).

Space limitations do not permit detailed discussion here of several PNNs that are known to be longer-period binaries. Two remarkable examples are (1) V651 Mon, the central star of NGC 2346, which is a 16-day spectroscopic binary with a highly variable light curve (Méndez and Niemela 1981; Jasniewicz and Acker 1986), and (2) IN Com (HD 112313), the central star of LT-5. The 
TABLE I: Photometrically Confirmed Binary PNNs $\left(P<1^{\mathrm{d}}\right)$

\begin{tabular}{ccrlc}
\hline Nebula & Central Star & Period & Type & Ref. \\
\hline Abell $41 \ldots$. & MT Ser & $2^{\mathrm{h}} 43^{\mathrm{m}}$ & Reflection & $(1)$ \\
DS $1 \ldots \ldots$. & KV Vel & $8^{\mathrm{h}} 34^{\mathrm{m}}$ & Reflection & $(2)$ \\
Abell $35 \ldots$. & $-22^{\circ} 3467$ & $\begin{array}{r}10^{\mathrm{h}} 23^{\mathrm{m}} \\
\text { or } 18^{\mathrm{h}} 23^{\mathrm{m}}\end{array}$ & Reflection? & $(3)$ \\
& & & \\
Abell $63 \ldots$. & UU Sge & $11^{\mathrm{h}} 10^{\mathrm{m}}$ & Eclipsing & $(4)$ \\
Abell $46 \ldots$. & V477 Lyr & $11^{\mathrm{h}} 19^{\mathrm{m}}$ & Eclipsing & $(5)$ \\
HFG $1 \ldots \ldots$ & & $13^{\mathrm{h}} 57^{\mathrm{m}}$ & Reflection & $(6)$ \\
K $1-2 \ldots \ldots$ & VW Pyx & $16^{\mathrm{h}} 05^{\mathrm{m}}$ & Reflection & $(7)$ \\
\hline
\end{tabular}

REFERENCES-(1) Grauer and Bond 1983; (2) Drilling 1985;

(3) Jasniewicz 1987; (4) Bond, Liller, and Mannery 1978;

(5) Bond 1985; (6) Grauer, Bond, Ciardullo, and Fleming $1987 a ;(7)$ Bond and Grauer $1987 a$.

optical spectrum of IN Com shows only a G-type star with rotationally broadened absorption lines and $\mathrm{H} \alpha$ emission (Bond 1985). Jasniewicz, Duquennoy, and Acker (1987) have found that the G star is itself a 2-day, double-lined spectroscopic pair, while a third body (presumably the hot component detected with IUE by Feibelman and Kaler 1983) orbits with a period suspected to be 41, 140, or 539 days.

Several additional PNNs have been reported to be short-period spectroscopic binaries by various authors (see references in Bond 1985 and Ritter 1986). Again, space does not permit a detailed discussion, but confirmation of these results, either through photometry or from additional spectroscopic observations, would be very important.

\section{LIGHT-CURVE ANALYSES}

Close-binary PNNs will provide astrophysical information about the component stars through analyses of their light curves and radial velocities. The most thorough analysis so far has been carried out by Drilling (1985) for KV Vel (LSS 2018), the central star of DS 1 . The primary star was found to have $T_{\text {eff }}=77,000 \mathrm{~K}$; the radii of the primary and secondary star are $0.2-0.3$ and $0.3-$ $0.4 R_{\odot}$, respectively, and the masses are 0.4-0.7 and 0.2-0.3 $M_{\odot}$. These results are completely consistent with a primary star that is in a pre-white-dwarf stage, and a lower-main-sequence secondary.

J. Kaluzny (in preparation) has analyzed the light curve of V477 Lyr, the eclipsing nucleus of Abell 46. The primary star is required to have $T_{\text {eff }}=$ $60,000 \mathrm{~K}$ to produce the observed reflection effect, and the radii of the primary and secondary are 0.2 and $0.6 R_{\odot}$. Except for an apparently somewhat more massive main-sequence secondary star, V477 Lyr appears to be quite similar to 
KV Vel. Both systems will become CVs when mass transfer from the secondary to the primary begins (several Gyr in the future).

\section{INCIDENCE OF CLOSE BINARIES AMONG PNNs}

Sufficient data are now available to permit an estimate of the frequency of occurrence of close-binary PNNs. Bond and Grauer (1987a) have listed 32 PNNs that did not show photometric variability when continuously monitored over intervals of several hours, and hence are unlikely to be close binaries. (The conspicuous heating effects described above imply that a close-binary PNN will show variations even if insufficiently inclined to show actual eclipses.) In a separate survey, Drummond (1980) monitored 17 PNNs (of which 6 are in common with the Bond-Grauer objects), and similarly found no short-term variability. Finally, Drilling (1985) mentions that KV Vel was the one variable found out of 3 objects that were monitored.

The observed incidence of close binaries among PNNs that have been monitored with photoelectric photometry is thus 7 out of 52 , or $14 \%$. Drummond's candidates emphasized the brighter PNs, while the Bond-Grauer objects have tended to be nuclei of low-surface-brightness PNs. Drilling observed 3 bright sdO stars surrounded by rather faint PNs. Therefore, the combined sample should be quite representative of the general population of PNNs.

The Bond-Grauer survey initially had a pre-selection for reported variability; for example, the nuclei of Abell 41, 46, and 63 were all suspected of variability by Abell (1966), and the variability of $\mathrm{K} 1-2$ was discovered on overlapping Palomar Sky Survey prints by Kohoutek (1964). However, the list of nonvariables is now large enough to begin to approach a magnitude-limited sample, and it should be noted that the remaining three binaries in Table I were discovered without any premonition of variability. The best estimate that can be made now is that about $10-15 \%$ of $P N N s$ are binaries with $P<1$ day. The binary fraction is, of course, even higher if longer-period systems, such as those mentioned above, are included, but these statistics are still very incomplete. Nevertheless, it is clear that binary-star processes are a significant production mechanism for planetary nebulae.

\section{BIRTH RATES}

Since we have argued that close-binary PNNs are the progenitors of CVs, it is important to ask whether the birth rates of the two types of objects are comparable.

The birth rate of PNs in the solar neighborhood has been the subject of a number of studies, of which a few recent ones are listed in Table II. The estimates are uncertain, principally because of lingering problems with the PN distance scale, and cover the range $1-8 \times 10^{-3} \mathrm{kpc}^{-3} \mathrm{yr}^{-1}$. The largest birth rate was obtained by Ishida and Weinberger (1987), who included several recently discovered, nearby, low-surface-brightness nebulae, and argue that the census of PNs is still incomplete even in the solar neighborhood.

Since it was concluded above that $10-15 \%$ of PNNs are close binaries, it follows that the birth rate for close-binary PNNs is $0.1-1.2 \times 10^{-3} \mathrm{kpc}^{-3} \mathrm{yr}^{-1}$. This may be compared with Patterson's (1984) estimated birth rate for CVs of all types of $0.007 \times 10^{-3} \mathrm{kpc}^{-3} \mathrm{yr}^{-1}$. This is likewise a rather uncertain estimate 
TABLE II: Local PN Birth Rate

\begin{tabular}{|c|c|}
\hline Reference & $\begin{array}{c}\text { Birth Rate } \\
\left(10^{-3} \mathrm{kpc}^{-3} \mathrm{yr}^{-1}\right)\end{array}$ \\
\hline Cahn \& Wyatt 1976........ & 5 \\
\hline Maciel $1981 \ldots \ldots \ldots \ldots \ldots$ & 2 \\
\hline Daub $1982 \ldots \ldots \ldots \ldots \ldots$ & 5 \\
\hline Mallik 1982 ............. & 2.4 \\
\hline Amnuel et al. $1984 \ldots \ldots \ldots$ & 4.6 \\
\hline Pottasch $1984 \ldots \ldots \ldots \ldots \ldots$ & 1.5 \\
\hline Drilling \& Schönberner 1985 & 1 \\
\hline Ishida \& Weinberger 1987 .. & 8 \\
\hline
\end{tabular}

because of the uncertain lifetimes of CVs, but the discrepancy of a factor of 15 or more between the close-binary PNN and CV birth rates is still uncomfortably large. However, a post-PNN origin for CVs could still be supported if one or both of the following were true:

1. Most of the immediate descendants of binary PNNs are accumulating as inconspicuous detached binaries that, within the Hubble time, have not yet initiated the mass transfer required to produce cataclysmic activity. Several of these "pre-cataclysmic" (or V471 Tau-type) binaries are known (see Bond 1985; Ritter 1986; and references therein). As discussed by Bond (1985), the majority of them have orbital periods so long that, in fact, several Gyr will be required for magnetic braking (Verbunt and Zwaan 1981) to bring the secondary stars into contact with their Roche lobes. Thus the active CVs observed at the present epoch could represent only the short-period tail of a considerably larger group of post-PNN binaries.

2. Surveys for CVs may still be quite incomplete, so that Patterson's birth rates could be seriously underestimated. Most of the nova-like CVs, for example, are not conspicuous objects; a ninth-magnitude nova-like variable was found just 3 years ago (Garrison et al. 1984). Moreover, it has recently been argued that classical novae spend large parts of their lives in very inconspicuous states of "hibernation" (Shara et al. 1986).

We conclude that the observed birth rates for close-binary PNNs and CVs are in sufficient agreement, given the uncertainties in both rates. It may be noted that Fleming, Liebert, and Green (1986) find a local white-dwarf birth rate of $0.5-0.75 \times 10^{-3} \mathrm{kpc}^{-3} \mathrm{yr}^{-1}$, a factor of 2-10 below the PN birth rate. They suggest that the census of white dwarfs in the solar neighborhood may be incomplete, because many white dwarfs are concealed in binary systems with main-sequence primaries. Such systems could include not only the pre-cataclysmic descendants of close-binary PNNs, but also much wider systems (like Sirius or Procyon).

\section{CATACLYSMIC PNNs?}

The discussion above presents a strong, but circumstantial, argument that CVs are descended from close-binary PNNs. The most direct evidence in support 
of this scenario would be the existence of PNs whose central stars are already mass-transferring CVs. This might be thought rather unlikely because of the fine tuning required to eject the common envelope at a time after the secondary's Roche lobe has shrunk down to the size of the secondary, but before actual coalescence of the double core. Astonishingly, however, recent work indicates that two such PNs with cataclysmic nuclei may actually exist:

1. The object $0623+71$ is a faint "bow-shock" nebula surrounding a 12thmag nova-like CV (Ellis, Grayson, and Bond 1984). We suggested that the nebula is an ejected shell resulting from an unrecorded nova outburst. Recently, however, Krautter, Klaas, and Radons (1987) have shown that the nebula does not have the large expansion velocity expected for a nova shell ( $V_{\exp }<100$ $150 \mathrm{~km} \mathrm{~s}^{-1}$ ), and have suggested that the shell is in fact a PN.

2. IRAS images of the region of the classical nova GK Per have recently been examined by Bode et al. (1987). GK Per was found to be surrounded by a large (radius $\sim 0.7 \mathrm{pc}$ ) infrared dust shell. The surface brightness is highest in the $100 \mu$ band, and a grain temperature of $22 \mathrm{~K}$ was derived. The $\mathrm{H}$ I mass was also derived from $21-\mathrm{cm}$ observations, and was found to be $\sim 0.6 M_{\odot}$, which is far too large to attribute to material ejected in prehistoric nova outbursts. The authors therefore argue that the shell is in fact an extremely old planetary nebula.

It may also be worth mentioning a remarkable series of visual observations of the nucleus of NGC 7662 made by Barnard (1908), who claimed variability over a range of more than 3 magnitudes. The behavior reported by Barnard (usually faint, occasionally bright) is reminiscent of the outbursts of dwarf novae. Modern CCD photometry (which would allow accurate subtraction of the nebular background) would be of interest.

\section{MORPHOLOGIES OF PNs WITH BINARY NUCLEI}

The morphologies of $\mathrm{PNs}$ ejected from close binaries through common-envelope interactions will be the subject of a forthcoming study by $\mathrm{M}$. Livio and the writer. Theoretical considerations (cf. Livio and Soker 1987) suggest that the initial shape of the PN will depend primarily upon the evolutionary stage of the primary star at the time it encounters its companion:

1. If the interaction occurs before the primary attains a very centrally condensed configuration, the timescale for orbital decay will drop to a value comparable to or less than the orbital period, and three-dimensional (nonspherical) effects will become important. The common envelope will be ejected preferentially in the orbital plane.

2. However, if the secondary star is not engulfed until the primary has reached an advanced (AGB) evolutionary stage, its highly centrally condensed configuration will make non-spherical effects less important. The ejected material will be less concentrated to the orbital plane.

During the subsequent evolution of a PN, a wind from the central star may lead to further modifications of the morphology, depending on the degree of density contrast (between the equatorial and polar regions of the nebula) that was set up during the envelope ejection (cf. Balick 1987). When a density contrast exists, the stellar wind will penetrate the nebula in the polar directions first.

In every case, the actual PNs known to contain close-binary nuclei have a non-spherical appearance. This is as expected for ejection from a binary system, 
but is of doubtful significance since $\sim 80 \%$ of all PNs have a non-spherical shape anyway (cf. Zuckerman and Aller 1986). The individual PNs with binary nuclei display a wide variety of morphologies. Abell 41 (illustrated by Grauer and Bond 1983) and Abell 63 have elliptical shapes, with the highest densities at both ends of the minor axes. The lower-density major axes probably represent an intermediate to late stage of "bubble penetration." NGC 2346 has a "butterfly" shape, which in Balick's scheme requires that a large density contrast was established in the ejection from the binary nucleus.

Several of the PNNs show evidence of interactions with the surrounding interstellar medium. Most of the nebular material in Abell 46 and HFG 1 lies on one side of the nucleus, suggesting ablation of the nebula due to its motion relative to the ISM. More extreme cases are Abell 35 and $0623+71$, which actually show a "bow-shock" morphology.

\section{THE PULSATING PNN K 1-16 AND RELATED OBJECTS}

While searching for binary PNNs, Grauer and Bond (1984) serendipitously discovered the only known pulsating central star, that of K 1-16 (DS Dra). Intensive photometric monitoring has shown $\mathrm{K} 1-16$ to be a multiperiodic, nonradial $g$-mode pulsator; numerous pulsation modes are simultaneously present and are concentrated near 1500 and $1700 \mathrm{sec}$ (Grauer and Bond 1987).

The optical spectrum of $\mathrm{K}$ 1-16 shows He II, C IV, and O VI features; no Balmer lines are present (Grauer and Bond 1984; Sion, Liebert, and Starrfield 1985). The spectroscopic and photometric properties of $K 1-16$ show that it is closely related to the four known pulsating GW Vir (PG 1159 - 035) white dwarfs. The GW Vir variables are extremely hot $\left(\sim 10^{5} \mathrm{~K}\right)$, hydrogen-deficient white dwarfs (Wesemael, Green, and Liebert 1985), whose pulsation periods generally lie in the range 400-600 sec (McGraw et al. 1979; Bond et al. 1984; Bond and Grauer 1987b). It is clear that they are more highly evolved than $\mathrm{K} 1-16$, because of their shorter pulsation periods and because they are not surrounded by observable PNs. (The strongest upper limit for a $P N$ around a GW Vir pulsator is at a level 60 times fainter than that of the Palomar Sky Survey, obtained for the pulsator PG 0122+200 by Reynolds 1987). K 1-16 may, in turn, be descended from the "O VI" class of hydrogen-deficient PNNs (Sion et al. 1985).

The pulsation mechanisms may be the same in both $\mathrm{K} 1-16$ and the GW Vir variables. Starrfield et al. $(1984,1985)$ have proposed that the mechanism is cyclical ionization of $\mathbf{C}$ and/or $O$ in the hydrogen-deficient envelope of the star. This suggestion is in accord with the spectroscopic appearance of the pulsators, although the detailed chemical compositions are still very uncertain (Liebert 1987). On the other hand, Iben (1984) and Wood and Faulkner (1986) have shown that hydrogen-deficient PNNs and their immediate descendants contain helium-burning shells. Kawaler et al. (1986) find that these shells are pulsationally unstable, suggesting that the $\epsilon$-mechanism is responsible for the $\mathrm{K} 1-16$ and GW Vir pulsations.

In a recent attempt to refine the location of the pulsational instability region in the HR diagram, Grauer et al. (1987b) showed that 14 additional hot, hydrogen-deficient PNNs and pre-white dwarfs do not pulsate. The 5 known pulsators have effective temperatures of $80,000-160,000 \mathrm{~K}$ and luminosities of 30-1000 $L / L_{\odot}(\log g=6-8)$. However, three non-pulsating members of the 
PG 1159 - 035 spectroscopic class defined by Wesemael et al. (1985) also lie in this region of the HR diagram, suggesting that the instability region is narrower than just indicated, or that the instability is critically dependent on some other stellar property, such as small changes in chemical composition. The null results would appear to cast doubt either on the presence of helium-burning shells in these objects, or on the $\epsilon$-mechanism for the pulsations, since for this mechanism all objects in this region of the HR diagram below $2000 L_{\odot}$ would be expected to pulsate (Kawaler et al. 1987). A recent photometric survey of PNNs for shortperiod pulsations by Hine and Nather (1987), as well as the Bond-Grauer binary PNN search described above, also failed to reveal any pulsators other than $\mathrm{K} \mathrm{1-16}$ itself.

Perhaps the greatest potential importance of $\mathrm{K} 1-16$ is that it may provide the evolutionary timescale of a PNN through measurement of its pulsational period change, $d P / d t$. For GW Vir itself, Winget et al. (1985) analyzed extensive photometric data to detect a period change on a timescale of $10^{6} \mathrm{yr}$. $\mathrm{K} \mathrm{1-16}$, which lies higher in the HR diagram than the GW Vir white dwarfs, should have a timescale more than an order of magnitude shorter. Unfortunately, the light variations of $K$ 1-16 are so complex, and the number of pulsation cycles per night so low, that it has not yet yielded to a similar analysis (Grauer and Bond 1987). It will probably be necessary to arrange continuous photometric monitoring from sites at different longitudes in order to make this important measurement.

A further important development in the study of these hot pulsators is the discovery that the modes in GW Vir appear to be spaced (nearly) uniformly in period (Kawaler $1987 a, b$ ). Since such uniform period spacings are expected for $g$-modes with the same degree $(l)$ and consecutive radial wave numbers $(n)$, it becomes possible to identify the pulsation modes. The observed periods of the modes then provide physical information about the star. Application of this technique to GW Vir provides an extraordinarily accurate (if the various assumptions are correct) mass determination of $0.60 \pm 0.02 M_{\odot}$. Moreover, slight departures from strictly uniform period spacings can provide information on the compositional stratification of the star (Kawaler 1987b). It would of course be of great interest to apply these techniques to the central star of K 1-16, but photometry from a single site may not be adequate for this formidable task.

\section{REFERENCES}

Abell, G.O. 1966, Ap. J., 144, 259.

Amnuel, P.R., Guseinov, O.H., Novruzova, H.I., and Rustamov, Yu.S. 1984, Ap. Space Sci., 107, 19.

Balick, B. 1987, Sky and Telescope, 73, 125.

Barnard, E.E. 1908, M.N.R.A.S., 68, 465.

Bode, M.F., Seaquist, E.R., Frail, D.A., Roberts, J.A., Whittet, D.C.B., Evans, A., and Albinson, J.S. 1987, Nature, in press.

Bond, H.E. 1976, Pub. A.S.P., 88, 192. 1985, in Cataclysmic Variables and Low-Mass X-Ray Binaries, eds. D.Q. Lamb and J. Patterson (Dordrecht: Reidel), p. 15.

Bond, H.E., and Grauer, A.D. 1987a, in IAU Colloq. No. 95, Second Conference on Faint Blue Stars, eds. A.G.D. Philip, D.S. Hayes, and J. Liebert (Schenectady: L. Davis Press), in press. 1987 b, Ap. J. (Letters), in press. 
Bond, H.E., Grauer, A.D., Green, R.F., and Liebert, J.W. 1984, Ap. J., $279,751$.

Bond, H.E., Liller, W., and Mannery, E.J. 1978, Ap. J., 223, 252.

Cahn, J.H., and Wyatt, S.P. 1976, Ap. J., 210, 508.

Daub, C.T. 1982, Ap. J., 260, 612.

Drilling, J.S. 1985, Ap. J. (Letters), 294, L107.

Drilling, J.S., and Schönberner, D. 1985, Astr. Ap., 146, L23.

Drummond, J.D. 1980, Ph.D. dissertation, New Mexico State University.

Ellis, G.L., Grayson, E.T., and Bond, H.E. 1984, Pub. A.S.P., 96, 283.

Feibelman, W.A., and Kaler, J.B. 1983, Ap. J., 269, 592.

Fleming, T.A., Liebert, J., and Green, R.F. 1986, Ap. J., 308, 176.

Garrison, R.F., Schild, R.E., Hiltner, W.A., and Krzeminski, W. 1984, Ap. J. (Letters), 276, L13.

Grauer, A.D., and Bond, H.E. 1983, Ap. J., 271, 259. 1984, Ap. J., 277, 211. 1987, in IAU Colloq. No. 95, Second Conference on Faint Blue Stars, eds. A.G.D. Philip, D.S. Hayes, and J. Liebert (Schenectady: L. Davis Press), in press.

Grauer, A.D., Bond, H.E., Ciardullo, R., and Fleming, T.A. 1987 a, Bull. A.A.S., $19,643$.

Grauer, A.D., Bond, H.E., Liebert, J., Fleming, T.A., and Green, R.F. 1987b, Ap. J., in press.

Hine, B.P., and Nather, R.E. 1987, in IAU Colloq. No. 95, Second Conference on Faint Blue Stars, eds. A.G.D. Philip, D.S. Hayes, and J. Liebert (Schenectady: L. Davis Press), in press.

Hoffleit, D. 1932, Harvard Bull., No. 887.

Iben, I. 1984, Ap. J., $277,333$.

Ishida, K., and Weinberger, R. 1987, Astr. Ap., 178, 227.

Jacoby, G.H. 1981, Ap. J., 244, 903.

Jasniewicz, G. 1987, in IAU Colloq. No. 95, Second Conference on Faint Blue Stars, eds. A.G.D. Philip, D.S. Hayes, and J. Liebert (Schenectady:

L. Davis Press), in press.

Jasniewicz, G., and Acker, A. 1986, Astr. Ap., 160, L1.

Jasniewicz, G., Duquennoy, A., and Acker, A. 1987, Astr. Ap., 180, 145.

Kawaler, S.D. 1987 a, in IAU Symp. No. 123, Advances in Helio- and Asteroseismology, eds. S. Christensen-Dalsgaard and S. Frandsen (Dordrecht: Reidel), in press.

Kawaler, S.D. 1987b, in IAU Colloq. No. 95, Second Conference on Faint Blue Stars, eds. A.G.D. Philip, D.S. Hayes, and J. Liebert (Schenectady:

L. Davis Press), in press.

Kawaler, S.D., Winget, D.E., Hansen, C.J., and Iben, I. 1986, Ap. J. (Letters), 306, L41.

Stellar Evolution, eds. S. Kwok and S. Pottasch (Dordrecht: Reidel), p. 403.

Kohoutek, L. 1964, Bull. Astron. Inst. Czech., 15, 161.

Krautter, J., Klaas, U., and Radons, G. 1987, Astr. Ap., 181, 373.

Liebert, J. 1987, in Stellar Pulsation, eds. A.N. Cox, W.M. Sparks, and S.G.

Starrfield (Berlin: Springer-Verlag), p. 342.

Livio, M., and Soker, N. 1987, submitted to Ap. J.

Maciel, W.J. 1981, Astr. Ap., 88, 406. 
Mallik, D.C.V. 1982, in IAU Symp. No. 103, Planetary Nebulae, ed. D.R. Flower (Dordrecht: Reidel), p. 424.

McGraw, J.T., Starrfield, S.G., Liebert, J., and Green, R. 1979, in IAU Colloq. No. 53, White Dwarfs and Variable Degenerate Stars, eds. H. Van Horn and V. Weidemann (Rochester: University of Rochester), p. 377.

Méndez, R.H., and Niemela, V.S. 1981, Ap. J., 250, 240.

Miller, J.S., Krzeminski, W., and Priedhorsky, W. 1976, IAU Circ., No. 2974.

Paczynski, B. 1976, in IAU Symposium No. 79, Structure and Evolution of CloseBinary Systems, eds. P. Eggleton, S. Mitton, and J. Whelan (Dordrecht: Reidel), p. 75.

Patterson, J. 1984, Ap. J. Suppl., 54, 443.

Pottasch, S.R. 1984, Planetary Nebulae (Dordrecht: Reidel).

Reynolds, R.J. 1987, Ap. J., 315, 234.

Ritter, H. 1976, M.N.R.A.S., 175, 279. 1986, Astr. Ap., 169, 139.

Shara, M.M., Livio, M., Moffat, A.F.J., and Orio, M. 1986, Ap. J., 311, 163.

Sion, E.M., Liebert, J., and Starrfield, S.G. 1985, Ap. J., 292, 471.

Starrfield, S., Cox, A.N., Kidman, R.B., and Pesnell, W.D. 1984, Ap. J., 281, 800.

293, L23. 1985, Ap. J. (Letters),

Verbunt, F., and Zwaan, C. 1981, Astr. Ap., 100, L7.

Wesemael, F., Green, R.F., and Liebert, J. 1985, Ap. J. Suppl., 58, 379.

Winget, D.E., Kepler, S.O., Robinson, E.L., Nather, R.E., and O'Donoghue, D. 1985, Ap. J., 292, 606.

Wood, P.R., and Faulkner, D.J. 1986, Ap. J., 307, 659.

Zuckerman, B., and Aller, L.H. 1986, Ap. J., 301, 772. 